quantitative approach proposed by Frey and coworkers may be extended and applied also to fluctuations of clinical symptoms, biomarkers, medication use and other endpoints used to monitor asthma.

Before lung function history indices can be implemented in our everyday clinical practice, more studies are required to improve the understanding of this new tool. For example, can the same measures of lung function history be applied to patients with asthma of different severity? The present study by Thamrin et $a l^{5}$ represents the first approach to this issue and, as mentioned, the data suggest that different indices may be needed in severe asthma than in mild to moderate asthma. However, the present study does not allow us to draw final conclusions on this issue. The studies of mild to moderate and severe asthma were made on two sets of data originating from two very different studies performed about 10 years apart and, as the authors point out, one limitation with the older study was that it did not use electronic diaries. Different inclusion criteria, different settings and a different course of the two trials also make the direct comparison of findings in these two cohorts difficult. There is therefore a need for confirmatory studies in well-phenotyped and coherent cohorts of patients with asthma of different severity and identical study protocols. It would be an added benefit if several biomarkers were determined repeatedly over a long time period in parallel with lung function data, symptoms and medication use. This would allow similar calculations of the history of biomarkers, symptoms and other outcomes, possibly adding information about the pathophysiology of asthma.

Considered together, the study of Thamrin et $a l^{5}$ raises excitement of future improvements both in clinical practice and mechanistic research. It seems that fluctuation analysis of data from handheld electronic spirometers should be a valuable new application to add to smartphones. This would make it possible to assess the value of lung function history measurements in research and also in ordinary clinical follow-up. For research, lung function fluctuation analysis may represent an important new key variable which, when integrated with other clinical and biological (genomic, transcriptomic, proteomic and metabolomic) data, may allow for better classification and phenotyping of asthma by the use of mathematical modelling in a systems biology approach. ${ }^{7}$ Such a better understanding of the pathology of asthmaespecially of severe, poorly-controlled or difficult-to-treat cases ${ }^{8}$-may facilitate development of new diagnostic methods and improve asthma care. Moreover, identification of key nodes in the complex network of inflammatory processes underlying asthma may result in discovery of new targets for effective therapeutic intervention.

Competing interests None
Provenance and peer review Not commissioned; internally peer reviewed.

Published Online First 19 August 2011

Thorax 2011;66:1019-1020.

doi:10.1136/thoraxjnl-2011-200847

\section{REFERENCES}

1. Global Initiative for Asthma. Global Strategy for Asthma Management and Prevention, 2008. http://www.ginasthma.com.

2. Reddel HK, Taylor DR, Bateman ED, et al. An official American Thoracic Society/European Respiratory Society statement: asthma control and exacerbations: standardizing endpoints for clinical asthma trials and clinical practice. Am J Respir Crit Care Med 2009;180:59-99.

3. Bousquet J, Mantzouranis E, Cruz AA, et al. Uniform definition of asthma severity, control and exacerbations: document presented for the World Health Organization Consultation on Severe Asthma. J Allergy Clin Immunol 2010;126:926-38.

4. Frey U, Brodbeck T, Majumdar A, et al. Risk of severe asthma episodes predicted from fluctuation analysis of airway function. Nature

2005; 438:667-70

5. Thamrin C, Nydegger R, Stern G, et al. Association between fluctuations in lung function and asthma control in two populations with differing asthma severity. Thorax 2011;66:1036-42.

6. Thamrin C, Zindel J, Nydegger R, et al. Predicting future risk of asthma exacerbations using individual conditional probabilities. J Allergy Clin Immunol 2011;127:1496-502.

7. Auffray C, Adcock IM, Chung KF, et al. An integrative systems biology approach to understanding pulmonary diseases. Chest 2010;137:1410-16.

8. Anon. The ENFUMOSA cross-sectional European multicentre study of the clinical phenotype of chronic severe asthma. European Network for Understanding Mechanisms of Severe Asthma. Eur Respir J 2003;22:470-7.

\title{
The challenges of quality improvement reports and the urgent need for more of them
}

\author{
Kieran McIntyre, ${ }^{1,2}$ Kaveh G Shojania ${ }^{2,3}$
}

Healthcare quality has received sustained attention since the release of To Err is Human by the US Institute of Medicine in

\footnotetext{
'Division of Respirology, St Michael's Hospital, Toronto, Ontario, Canada; ' ${ }^{2}$ Department of Medicine, University of Toronto, Toronto, Ontario, Canada; ${ }^{3}$ Sunnybrook Health Sciences Centre, the University of Toronto, Centre for Patient Safety, Toronto, Ontario, Canada
}

Correspondence to Dr Kieran McIntyre, Division of Respirology, St. Michaels Hospital, Rm 6-037, Toronto, ON M5B 1W8, Canada; mcintyrek@smh.ca late $1999 .{ }^{1}$ This report captured widespread interest with the oft-quoted estimate that medical errors annually cause 44000-98000 deaths in US hospitals alone. This period also coincided with publication of 'An organisation with a memory', ${ }^{2}$ which described the scale and nature of serious failures in the UK National Health Service.

A widely accepted definition describes quality as the degree to which health services for individuals and populations increase the likelihood of desired health outcomes and are consistent with current professional knowledge. ${ }^{3} 4$ This definition further characterised quality in terms of six dimensions: safety, effectiveness, patient centeredness, timeliness, efficiency and equitability.

Numerous studies document major shortcomings in each of these dimensions across a range of clinical settings. ${ }^{3}$ One illustrative study ${ }^{5}$ showed that only $55 \%$ of Americans with chronic medical conditions received basic aspects of acute, chronic and preventive care. ${ }^{5}$ For example, only $50 \%$ of patients with asthma received chronic inhaled corticosteroids and a similarly low percentage of patients with chronic obstructive pulmonary disease (COPD) received influenza vaccination. These major shortfalls in effective healthcare do not simply reflect access issues, as comparable data from Canada (with universal public healthcare) show that only 
$56 \%$ of patients with COPD had undergone spirometry as recommended by guidelines and only $34 \%$ received guidelineconcordant treatment. ${ }^{6}$ Given that COPD will become the third leading cause of death by $2030^{7}$ and represents the one common cause of death for which mortality rates continue to climb, we must improve adherence to evidence-based aspects of COPD management. ${ }^{8}$

\section{ADDRESSING QUALITY PROBLEMS}

Quality improvement (OI) is a science ${ }^{9}$ and includes numerous distinct strategies for changing patient and provider behaviour, as well as redesigning systems of care-audit and feedback, case management, support for self-management, patient registries and computerised decision support to name just a few. ${ }^{10-12}$ But, the single most basic approach involves iterative cycles of outcome measurement, identification of problems, implementation of potential solutions and repeated measurement. ${ }^{13}$

The positive impact of such cycles of continuous $\mathrm{OI}$ in pulmonary medicine has been nowhere as evident as under the direction of the American Cystic Fibrosis Foundation Patient Registry and its Therapeutic Development Network. In this issue, Drs Quon and Goss provide a review of the huge impacts these initiatives have had on the lives of patients with cystic fibrosis. ${ }^{14}$ The overriding principle has been transparency, with all participating centres committed to reporting their results to clinicians and patients.

The American Cystic Fibrosis Foundation Patient Registry has evolved over 45 years from a few basic measures of the natural history of disease to over 300 variables for some 26000 patients, detailing aspects of management, pulmonary functional status, laboratory data and clinical outcomes, as well patients' (or their parents') assessments of the quality of care received. This engagement in transparently measuring and improving care has been associated with continued improvements in outcomes, including an increase in life expectancy from 27 years in 1989 to 36 years in 2009. ${ }^{14}$

\section{CHALLENGES IN REPORTING IMPROVEMENT EFFORTS}

We urgently need more such successful improvement initiatives in pulmonary medicine. That said, reporting the methods and results of OI initiatives differs in important ways from reports of traditional clinical research. OI reports tend to address messier problems, involve more complex interventions and require far greater attention to context (table 1).

The 'messiness' of problems in OI reflects their broader scope and focus on routine care, rather than the idealised setting of a clinical trial. For instance, a clinical trial might address the question: Does such-and-such drug improve the following specific clinical outcome for patients with COPD? An improvement project, by contrast, might ask: Can we improve outcomes for patients with COPD by reorganising our referral and scheduling processes to ensure timely access and better coordination between specialists and general practitioners? This example illustrates not just the 'messiness' problem, but also the intrinsic complexity of the interventions. When reporting a clinical trial, the intervention typically requires scant description because its components are well understood: a drug with known ingredients, administered according to a specified regimen, with such-and-such processes related to follow-up assessment. By contrast, reporting changes to a clinic's referral and scheduling processes requires detailed description, because none of the changes involve ubiquitous or well-understood ingredients and actions.

Messy as the problems of OI are and complex as the associated interventions can be, the crucial role of context in reporting and interpreting improvement initiatives adds a unique dimension that has received increasing attention. ${ }^{15}$ Potentially relevant contextual factors include external environmental influences (eg, regulatory requirements, payment systems, media attention) and numerous organisational features, such as resources, technologies, staffing, institutional culture and baseline quality, among others.

In interpreting a clinical trial, we do not need to know the psychological or institutional motivations that gave rise to the trial. ("My father suffered with COPD for many years and the head of my department encouraged me to focus on this promising new drug.') We do not require such details because, except in the case of commercial interests, they have no bearing on the conduct or interpretation of the research. With OI, however, stating that 'our hospital undertook this initiative after media reports of poor outcomes' and 'the president of the hospital championed this improvement project' suggests factors that may have directly affected the project's success-staff motivation, executive support for necessary policy changes and provision of resources.

Table 1 Challenges that distinguish quality improvement (Ol) from traditional clinical research

\begin{tabular}{|c|c|c|}
\hline & Typical clinical research & Typical 0l \\
\hline $\begin{array}{l}\text { 'Messier problems' in } 0 \text { l } \\
\text { Outcomes and goals less well defined, } \\
\text { settings less controlled }\end{array}$ & $\begin{array}{l}\text { In the highly controlled setting of a clinical trial, } \\
\text { what effect do drugs } A, B \text { and } C \text { have on } X, Y \text { and } \\
Z \text { well-defined outcomes in the following highly } \\
\text { selected patient population? }\end{array}$ & $\begin{array}{l}\text { In the setting of routine care, does reorganising the } \\
\text { following } A, B \text { and } C \text { aspects of care delivery significantly } \\
\text { improve } X, Y \text { and } Z \text { outcomes related to quality of } \\
\text { care (usually not nearly as easily defined or measured } \\
\text { as standard clinical outcome) in an unselected population? }\end{array}$ \\
\hline $\begin{array}{l}\text { Complex interventions } \\
\text { Ol interventions require much more description } \\
\text { to understand and to permit replication } \\
\text { Who performed what functions in the 'multidisciplinary } \\
\text { team'? What exactly did the electronic registry do? } \\
\text { Who were the 'project champions' and how } \\
\text { were they selected? }\end{array}$ & $\begin{array}{l}\text { Patients in the intervention group received drugs } \\
A, B \text { and } C \text { at such-and-such doses over the } \\
\text { specified time period; the control group } \\
\text { received placebo }\end{array}$ & $\begin{array}{l}\text { Intervention included multidisciplinary teams, an electronic } \\
\text { patient registry to track key aspects of disease } \\
\text { management and generate reminders to patients and } \\
\text { providers, project champions to help engage clinicians } \\
\text { and educational conferences held. Control group } \\
\text { received usual care, which consisted of } x, y \text { and } \\
z \text { processes of care }\end{array}$ \\
\hline $\begin{array}{l}\text { Context plays a crucial role What motivated the } \\
\text { intervention? } \\
\text { What institutional features provided direct or indirect } \\
\text { support for the intervention? } \\
\text { What internal and external incentives might have } \\
\text { affected the behaviours of participating } \\
\text { clinicians and patients? }\end{array}$ & $\begin{array}{l}\text { The intervention took place in a teaching hospital } \\
\text { with } \mathrm{N} \text { beds and access to such-and-such support } \\
\text { services and technologies }\end{array}$ & $\begin{array}{l}\text { The intervention was developed after a critical incident } \\
\text { resulted in a patient death and received attention in } \\
\text { local news media } \\
\text { Senior management supports the intervention through } \\
\text { periodic messages and facilitating necessary changes in } \\
\text { the clinic's information systems } \\
\text { A national system for public reporting of institutional } \\
\text { performance included outcomes affected by } \\
\text { this intervention }\end{array}$ \\
\hline
\end{tabular}


The general issues illustrated in table 1 encompass numerous specific factors potentially relevant to the interpretation of OI research. The SQUIRE (Standards for QUality Improvement Reporting Excellence) statement provides a checklist of 19 items that authors should consider when reporting QI studies. Most items are common to all scientific reporting, but many have been modified to reflect the unique nature of improvement work. ${ }^{16}$ For instance, the Introduction should include not just a description of relevant background literature but also an explicit description of the local problem that gave rise to the initiative. And the Methods should include not just the usual sections on study design, outcomes of interest and analytic methods, but also describe planning and implementation of the intervention (eg, why specific components were chosen, how they were expected to work).

The importance of this SQUIRE framework can be seen when applied to the published report of a single centre's experience to improve clinician adherence to best practice guidelines for asthma and COPD. ${ }^{17}$ The intervention consisted of developing a set of evidence-based performance indicators, use of an electronic medical record to support automated generation of performance reports, discussion of division-level reports at regular faculty meetings and quarterly provision of individual performance reports to each faculty member.

Using the SQUIRE checklist, one would include not just the basic description of the academic respirology division in which in the intervention occurred, but what specific local interest motivated the effort. One would also want to report some detail about the amount of effort required to use the electronic medical record system to provide usable performance reports. And why choose performance reports as the intervention? Unless the main issues underlying the targeted problems all fell under physicians' control, feeding back performance reports to physicians would serve little purpose. Finally, the attitudes of the division's leaders and faculty members would help understand their receptivity to the performance reports.

This specific paper ${ }^{17}$ reports information recommended in SQUIRE to a variable degree. However, our point lies not in critiquing this paper, but rather in pointing out the degree to which using the SQUIRE checklist (available at http://www.squire-statement.org/assets/ pdfs/SQUIRE_guidelines_table.pdf) facilitates interpretation of the study's results and informs readers' decisions of whether or not such an intervention might work in their practice settings. ${ }^{15}$

Like the CONSORT statement for the reporting of randomised trials, ${ }^{18}$ the goal of SQUIRE lies not just in improved reporting, but also in improved design. One would not want clinical trialists to find out about concealed allocation and blinding only at the stage of consulting CONSORT to write up their results. Similarly, recognising the importance of issues covered in SQUIRE will enhance the success of OI research, not just its publication. For instance, the exhortation to report details such as collaboration with major patient advocacy groups and the focus on transparent, detailed reporting of outcomes, as occurred with initiatives in cystic fibrosis, ${ }^{14}$ also suggests the importance of considering such features in other OI initiatives for chronic illnesses (eg, COPD, diabetes, congestive heart failure, asthma). These specific components may not prove essential in all cases, but the general model followed in cystic fibrosis should serve as a call to arms for others to improve patient care and SQUIRE provides a framework for enhancing both the rigour and the reporting of all such efforts.

\section{Competing interests None.}

Provenance and peer review Commissioned; internally peer reviewed.

Published Online First 19 August 2011

Thorax 2011;66:1020-1022.

doi:10.1136/thoraxjnl-2011-200853

\section{REFERENCES}

1. Kohn L, Corrigan JM, Donaldson M. To Err Is Human: Building a Safer Health System. Washington, DC: National Academy Press, 2000.

2. Donaldson L. An organisation with a memory. Clin Med 2002;2:452-7.

3. Institute of Medicine (US). Crossing the quality chasm: A New Health System for the 21st Century. Committee on Quality of Health Care in America. Washington, DC: National Academy Press, 2001:337pp

4. Berwick DM. A user's manual for the IOM's 'quality chasm' report. Health Aff (Millwood) 2002;21:80-90.

5. McGlynn EA, Asch SM, Adams J, et al. The quality of health care delivered to adults in the United States. N Engl J Med 2003;348:2635-45.

6. Bourbeau J, Sebaldt RJ, Day A, et al. Practice patterns in the management of chronic obstructive pulmonary disease in primary practice: the CAGE study. Can Respir J 2008;15:13-19.

7. WHO. World Health Report. http://www.who.int/ respiratory/copd/World_Health_Statistics_2008/en/ index.html2008.

8. Rabe KF, Hurd S, Anzueto A, et al. Global strategy for the diagnosis, management, and prevention of chronic obstructive pulmonary disease: gold executive summary. Am J Respir Crit Care Med 2007; 176:532-55.

9. Berwick DM. The science of improvement. JAMA 2008;299:1182-4.

10. Shojania KG, Grimshaw JM. Evidence-based quality improvement: the state of the science. Health Aff (Millwood) 2005;24:138-50.

11. Shojania KG, Ranji SR, McDonald KM, et al. Effects of quality improvement strategies for type 2 diabetes on glycemic control: a meta-regression analysis. JAMA 2006;296:427-40.

12. Bravata DM, Gienger AL, Holty JE, et al. Quality improvement strategies for children with asthma: a systematic review. Arch Pediatr Adolesc Med 2009:163:572-81.

13. Berwick DM. Continuous improvement as an ideal in health care. N Engl J Med 1989;320: 53-6.

14. Quon BS, Goss CH. A story of success: continuous quality improvement in cystic fibrosis care in the USA. Thorax 2011;66:1106-8.

15. Stevens DP, Shojania KG. Tell me about the context, and more. BMJ Qual Saf 2011;20:557-9.

16. Ogrinc G, Mooney SE, Estrada C, et al. The SQUIRE (Standards for QUality Improvement Reporting Excellence) guidelines for quality improvement reporting: explanation and elaboration. Qual Saf Health Care 2008;17(Suppl 1):i13-32.

17. Roberts DH, Gilmartin GS, Neeman N, et al. Design and measurement of quality improvement indicators in ambulatory pulmonary care: creating a "culture of quality" in an academic pulmonary division. Chest 2009;136:1134-40.

18. Schulz KF, Altman DG, Moher D, et al. 2010 statement: updated guidelines for reporting parallel group randomised trials. BMJ 2010;340:c332. 